\title{
EXISTENCE AND UNIQUENESS OF SOLUTIONS OF NONLINEAR SYSTEMS OF CONDUCTIVE-RADIATIVE HEAT TRANSFER EQUATIONS *
}

\author{
C. T. KELLEY ${ }^{\dagger}$
}

\begin{abstract}
We prove an existence and uniqueness results for a system of nonlinear integrodifferential equations that model steady-state combined radiative-conductive heat transfer. Our approach uses two different formulations of the system as a compact fixed-point problem. One formulation, which has been used in numerical work, is used for uniqueness and a new one is used for the existence proof.
\end{abstract}

Key words. radiative-conductive heat transfer, compact fixed point problems, existenceuniqueness

AMS(MOS) subject classifications. 45G10, 45K05, 82A70,

1. Introduction. In [9] and [5] models for coupled radiative-conductive heat transport are discussed. These models can be expressed as nonlinear systems of transport and diffusion equations. The purpose of this paper is to prove existence and uniqueness for these systems. In the interests of simplicity, we consider only isotropic scattering, homogeneous media, and Dirichlet boundary conditions. Our approach may be extended directly to the more complicated cases involving reflecting boundary conditions, inhomogeneous media, and anisotropic scattering, using the methods described in [3], [7], and [4].

In this introductory section we describe the system of equations we seek to solve. In $\S 2$ we reformulate the system as a compact fixed point problem in two ways. The first approach has been the basis for numerical results [9], [8]. The second, new in this paper, is applied to prove existence and uniqueness results in $\S 3$ and $\S 4$.

We consider the normalized form of the equations as described in [5], [9], and [8]. The radiative transport equation is

$$
\mu \frac{\partial \psi}{\partial x}(x, \mu)+\psi(x, \mu)=\frac{c}{2} \int_{-1}^{1} \psi\left(x, \mu^{\prime}\right) d \mu^{\prime}+(1-c) \Theta^{4}(x),
$$

for $x \in(0, \tau)$ with boundary conditions

$$
\psi(0, \mu)=\Theta_{l}^{4}, \mu>0 ; \psi(\tau, \mu)=\Theta_{r}^{4}, \mu<0 .
$$

We assume throughout that $c \leq 1$.

The temperature $\Theta$ satisfies the diffusion equation.

$$
\frac{\partial^{2} \Theta}{\partial x^{2}}=Q(x),
$$

for $x \in(0, \tau)$ with boundary conditions

$$
\Theta(0)=\Theta_{l}, \Theta(\tau)=\Theta_{r},
$$

\footnotetext{
* This document was printed on February 19, 1995.

$\dagger$ North Carolina State University, Center for Research in Scientific Computation and Department of Mathematics, Box 8205, Raleigh, N. C. 27695-8205, USA. This research was supported by National Science Foundation grant \#DMS-9321938. Computing activity was partially supported by an allocation of time from the North Carolina Supercomputing Center.
} 
and coupling to the radiative transport by

$$
Q(x)=\frac{1}{2 N_{c}} \frac{d}{d x} \int_{-1}^{1} \mu^{\prime} \psi\left(x, \mu^{\prime}\right) d \mu^{\prime} .
$$

In (1.5) $N_{c}$ is the conduction to radiation parameter [5].

2. Compact Fixed Point Problem. In this section we show how existence of a sufficiently regular solution of the system in $\$ 1$ can be related to existence of a solution $\Theta$ of a compact fixed point problem in two ways. We apply one of these formulations to prove regularity of solutions and will use the other to prove existence in $\xi 3$.

Let $\Theta \in C[0, \tau]$ be given with $\Theta(0)=\Theta_{l}$ and $\Theta(\tau)=\Theta_{r}$. Define $\psi$ by (1.1) and (1.2). $\psi$ exists since $c<1$. It is well known [2], [1], that the flux

$$
f(x)=\frac{1}{2} \int_{-1}^{1} \psi\left(x, \mu^{\prime}\right) d \mu^{\prime}
$$

satisfies

$$
f(x)-c \int_{0}^{\tau} k(x, y) f(y) d y=g(x)
$$

where

$$
\begin{gathered}
k(x, y)=\frac{1}{2} E_{1}(|x-y|), \\
E_{1}(|x-y|)=\int_{0}^{1} \exp (-|x-y| / \nu) \frac{d \nu}{\nu},
\end{gathered}
$$

and

$$
\begin{aligned}
g(x) & =\frac{\Theta_{l}^{4}}{2} \int_{0}^{1} \exp (-x / \nu) d \nu \\
& +\frac{\Theta_{r}^{4}}{2} \int_{0}^{1} \exp (-(\tau-x) / \nu) d \nu+\frac{(1-c)}{2} \int_{0}^{\tau} E_{1}(|x-y|) \Theta^{4}(y) d y
\end{aligned}
$$

We let $\mathcal{K}$ denote the integral operator defined for all $u \in L^{1}(0, \tau)$ by

$$
(\mathcal{K} u)(x)=\int_{0}^{\tau} k(x, y) u(y) d y
$$

It is known [1] that if $\tau<\infty$ then $\mathcal{K}$ is a compact operator from $L^{1}[0, \tau]$ to $C[0, \tau]$ and $I-c \mathcal{K}$ is a bounded operator on any $L^{p}[0, \tau]$ for $1 \leq p \leq \infty$.

We define

$$
\phi\left(x: \Theta_{l}, \Theta_{r}\right)=\frac{\Theta_{l}^{4}}{2} \int_{0}^{1} \exp (-x / \nu) d \nu+\frac{\Theta_{r}^{4}}{2} \int_{0}^{1} \exp (-(\tau-x) / \nu) d \nu
$$

and express $f$ in terms of $\Theta$ by

$$
f=(1-c)(I-c \mathcal{K})^{-1} \mathcal{K} \Theta^{4}+(I-c \mathcal{K})^{-1} \phi\left(\cdot: \Theta_{l}^{4}, \Theta_{r}^{4}\right)
$$


In Appendix A we show that the formal exchange of differentiation and integration

$$
\frac{d}{d x} \int_{-1}^{1} \mu^{\prime} \psi\left(x, \mu^{\prime}\right) d \mu^{\prime}=\int_{-1}^{1} \mu^{\prime} \frac{\partial}{\partial x} \psi\left(x, \mu^{\prime}\right) d \mu^{\prime}
$$

is valid for $0<x<\tau$. Hence, using (1.1),

$$
\begin{aligned}
\frac{d}{d x} \int_{-1}^{1} \mu^{\prime} \psi\left(x, \mu^{\prime}\right) d \mu^{\prime} & =-\int_{-1}^{1} \psi\left(x, \mu^{\prime}\right) d \mu^{\prime}+2 c f(x)+2(1-c) \Theta^{4}(x) \\
& =2(c-1) f(x)+2(1-c) \Theta^{4}(x) .
\end{aligned}
$$

Therefore, setting $\alpha=(1-c) / N_{\ell}$, we can use (2.7) and (1.5) to express $Q$ as

$$
\begin{aligned}
Q(x) & =\frac{(c-1) f(x)+(1-c) \Theta^{4}(x)}{N_{c}} \\
& =\alpha\left(\Theta^{4}-f\right)
\end{aligned}
$$

for $0<x<\tau$. Using $(2.5)$ we have

$$
Q=\alpha\left[(I-c \mathcal{K})^{-1}(I-\mathcal{K}) \Theta^{4}-(I-c \mathcal{K})^{-1} \phi\right]
$$

where $\phi$ is given by (2.4). Continuity of $\Theta$ and $\phi$ therefore imply continuity of $Q$.

We let $\mathcal{G}$ be the solution map for the negative Laplacian with homogeneous boundary conditions, i. e.

$$
-(\mathcal{G} u)_{x x}=u ; \quad \mathcal{G}(u)(0)=\mathcal{G}(u)(\tau)=0 .
$$

We define the fixed point map $\mathcal{F}$, which depends parametrically on $\Theta_{l}, \Theta_{r}$ and $c$ by

$$
\begin{aligned}
\mathcal{F}(\Theta) & =-\alpha \mathcal{G}\left((I-\mathcal{K})(I-c \mathcal{K})^{-1} \Theta^{4}-(I-c \mathcal{K})^{-1} \phi\right) \\
& +\frac{\Theta_{l}(\tau-x)+\Theta_{r} x}{\tau} .
\end{aligned}
$$

Note that if we define

$$
\mathcal{L}=-\mathcal{G}(I-\mathcal{K})(I-c \mathcal{K})^{-1} \text { and } \beta=\alpha \mathcal{G}(I-c \mathcal{K})^{-1} \phi+\tau^{-1}\left(\Theta_{l}(\tau-x)+\Theta_{r} x\right)
$$

we may write

$$
\mathcal{F}(\Theta)=\alpha \mathcal{L}\left(\Theta^{4}\right)+\beta .
$$

This formulation is attractive for numerical purposes in that evaluation of the fixed point map does not require the solution of a nonlinear equation. This is essentially the formulation used in the numerical studies reported in [9] and [8].

We can obtain a regularity result directly from (2.10) or (2.11).

Theorem 2.1. Let parameters $\Theta_{l}, \Theta_{r}$ and $c$ be given with $0 \leq c \leq 1$. Let $\Theta \in C[0, \tau]$ be a fixed point of $\mathcal{F}$, where $\mathcal{F}$ is given by $(2.10)$. Then $\Theta \in C^{2}[0, \tau]$ and if $\psi$ is a solution of the boundary problem given by $(1.1)$ and $(1.2)$, the pair $(\Theta, \psi)$ is a solution of the system (1.1), (1.2), (1.3), (1.4).

Proof. Note that $\mathcal{F}$ is a continuous map from $C[0, \tau]$ to $C^{2}[0, \tau]$. Hence, if $\Theta$ is a fixed point of $\mathcal{F}$, then $\Theta$ satisfies (1.3) with $Q$ given by (2.8). 
One can proceed from (2.8) in a different way. We define $F\left(\Theta^{4}\right)=f$, where $f$ is the solution of $(2.2) . F$ is a completely continuous map on $C[0, \tau]$. With this notation, $\mathcal{F}(\Theta)$ is the solution $T$ to the linear boundary value problem

$$
T^{\prime \prime}=\alpha\left(\Theta^{4}-F\left(\Theta^{4}\right)\right) ; T(0)=\Theta_{l}, T(\tau)=\Theta_{r} .
$$

Our alternative formulation is to define $\mathcal{T}(\Theta)$ to be the unique nonnegative solution $T$ of the nonlinear problem

$$
T^{\prime \prime}=\alpha\left(T^{4}-F\left(\Theta^{4}\right)\right) ; T(0)=\Theta_{l}, T(\tau)=\Theta_{r} .
$$

Our second compact fixed problem formulation is

$$
\Theta=\mathcal{T}(\Theta)
$$

At times in our analysis the dependence of $\mathcal{T}$ and $F$ on the boundary data $\Theta_{l}$ and $\Theta_{r}$ will be important. When that is so we may make that dependence explicit by writing $F\left(\Theta^{4}, \Theta_{l}, \Theta_{r}\right)$ and $\mathcal{T}\left(\Theta, \Theta_{l}, \Theta_{r}\right)$. We must prove, of course, that (2.12) has a unique nonnegative solution, which we do in the next section.

3. Existence of Solutions. Our existence results are based on the fixed point problem (2.13). We will apply the Schauder fixed point theorem [6] to the map $\mathcal{T}$. To do this we must first show that the map $\mathcal{T}$ is defined and completely continuous. We define

$$
\Theta_{-}=\min \left(\Theta_{l}, \Theta_{r}\right) \text { and } \Theta_{+}=\max \left(\Theta_{l}, \Theta_{r}\right) .
$$

We then show that $\mathcal{T}$ maps the convex and bounded set

$$
D=\left\{u \in C[0, \tau] \mid \Theta_{-} \leq u \leq \Theta_{+}\right\}
$$

into itself.

3.1. Properties of the map E. We begin with several results on (3.3). With these results in hand we will be able to express $\mathcal{T}$ as a composition of two nonlinear maps, $F$ and $E$, where $F$ takes $D$ to $\underline{D}$, where

$$
\underline{D}=\left\{f \in C[0, \tau] \mid \Theta_{-}^{4} \leq f \leq \Theta_{+}^{4}\right\} .
$$

and $E: \underline{D} \rightarrow D$ is defined as follows. For $f \in \underline{D}$ and

$$
\Theta_{-} \leq \Sigma_{l}, \Sigma_{r} \leq \Theta_{+}
$$

define $T=E\left(f, \Sigma_{l}, \Sigma_{r}\right)$ as the solution to

$$
T^{\prime \prime}=\alpha\left(T^{4}-f\right) ; T(0)=\Sigma_{l}, T(\tau)=\Sigma_{r} .
$$

When we write $E(f)$ it will be understood that $\Sigma_{l}=\Theta_{l}$ and $\Sigma_{r}=\Theta_{r}$. Our first results use standard methods [6] to show that (3.3) has a unique nonnegative solution $T \in D$ satisfies monotonicity properties with respect to its data. Hence $E$ is well defined.

Lemma 3.1. Let $u_{r}, u_{l} \geq 0,[a, b]$ a bounded interval, $\phi$ a strictly increasing and bounded function defined on $(-\infty, \infty)$. Let $f \in C[a, b]$ be such that

$$
\phi\left(\min \left(u_{l}, u_{r}\right)\right) \leq f \leq \phi\left(\max \left(u_{l}, r_{r}\right)\right)
$$


everywhere in $[a, b]$. Then the boundary value problem

$$
u^{\prime \prime}=\phi(u)-f ; u(a)=u_{l}, u(b)=u_{r}
$$

has a solution $u$. Moreover any solution $u$ is continuous and satisfies

$$
\min \left(u_{l}, u_{r}\right) \leq u \leq \max \left(u_{l}, u_{r}\right)
$$

Proof. Let $\mathcal{G}$ be the inverse of $-d^{2} / d x^{2}$ with homogeneous Dirichlet boundary conditions on $[a, b]$. The boundary value problem $(3.5)$ is equivalent to the compact fixed point problem

$$
u=\mathcal{G}(f-\phi(u))+\frac{u_{l}(b-x)+u_{r}(x-a)}{b-a}
$$

which has a solution in $C[a, b]$ by the boundedness of $\phi$ and the Schauder fixed point theorem. Clearly any such solution is in $C^{2}$.

Now let $u_{-}=\min \left(u_{l}, u_{r}\right)$ and $u_{+}=\max \left(u_{l}, u_{r}\right)$. If $u(\bar{x})<u_{-}$for some $x \in[a, b]$ then there is an interval $[c, d]$ such that $u<u_{-}$on $(c, d)$ and $u(c)=u(d)=u_{-}$. Then (3.4) implies that $u^{\prime \prime}<0$ and hence $u$ is concave on $[c, d]$. This implies that $u(\bar{x}) \geq u_{-}$, a contradiction. Hence $u \geq u_{-}$on $[a, b]$. Similarly $u \leq u_{+}$everywhere on $[a, b]$. ㅁ

As an easy consequence, we see that (3.3) has a unique solution in $D$ for $f \in \underline{D}$.

ThEOREM 3.2. Let $f \in \underline{D}$. Then (3.3) has a unique nonnegative solution $T \in \underline{D}$. Proof. Define

$$
\phi(\Theta)= \begin{cases}\Theta_{-}^{4}-\frac{\left(\Theta_{-}-\Theta\right)}{1+\left(\Theta_{-}-\Theta\right)}, & \Theta<\Theta_{-} \\ \Theta^{4}, & \Theta_{-} \leq \Theta \leq \Theta_{+} \\ \Theta_{+}^{4}+\frac{\left(\Theta-\Theta_{+}\right)}{1+\left(\Theta_{-} \Theta_{+}\right)}, & \Theta>\Theta_{+} .\end{cases}
$$

$\phi$ is strictly increasing, bounded, and

$$
\begin{array}{cc}
\phi(T)<\Theta_{-}^{4} & \text { if } T<\Theta_{-} \\
\phi(T)>\Theta_{+}^{4} & \text { if } T>\Theta_{+}
\end{array}
$$

Now, consider the equation

$$
T^{\prime \prime}=\alpha(\phi(T)-f), T(0)=\Theta_{l}, T(\tau)=\Theta_{r} .
$$

Since $f \in \underline{D}$, (3.8) has a solution in $D$ by Lemma 3.1. Since $T \in D, \phi(T)=T^{4}$ and (3.8) is equivalent to $(2.12)$.

To prove the uniqueness assertion, let $T$ and $S$ be two nonnegative solutions of (3.3). Then $w=T-S$ solves the boundary value problem

$$
w^{\prime \prime}=\alpha p w ; w(0)=w(\tau)=0 .
$$

The function

$$
p=\left(T^{4}-S^{4}\right) /(T-S)=(T+S)\left(T^{2}+S^{2}\right) \geq 0
$$


because $T, S \geq \Theta_{-} \geq 0$. A convexity argument similar to those above implies that $w=0$. Hence (3.3) has a unique nonnegative solution. This completes the proof. $\square$

At this point we have shown that $E(f)$ is well defined. An argument similar to the uniqueness part of the proof of Theorem 3.2 proves

Corollary 3.3. Let $f \geq g \geq 0, T_{l} \geq S_{l} \geq 0, T_{r} \geq S_{r} \geq 0$, and $\alpha \geq 0$. Let $T$ and $S$ solve the boundary value problems

$$
T^{\prime \prime}=\alpha\left(T^{4}-f\right) ; T(a)=T_{l}, T(b)=T_{r} \text { and } S^{\prime \prime}=\alpha\left(S^{4}-g\right) ; S(a)=S_{l}, S(b)=S_{r} .
$$

Then $T \geq S$ in $[a, b]$. This can be restated in terms of $E\left(f, \Sigma_{l}, \Sigma_{r}\right)$ as

Lemma 3.4. Let $c \in[0,1], \tau \in(0, \infty), f, g \in \underline{D}, f \leq g$ and

$$
\begin{aligned}
& \Theta_{-} \leq \Sigma_{l} \leq \Theta_{l} \leq \Theta_{+} \\
& \Theta_{-} \leq \Sigma_{r} \leq \Theta_{r} \leq \Theta_{+}
\end{aligned}
$$

then

$$
E\left(f, \Sigma_{l}, \Sigma_{r}\right) \leq E\left(g, \Theta_{l}, \Theta_{r}\right)
$$

for all $x \in[0, \tau]$. results.

We close our discussion of $E$ with a continuity, differentiability, and compactness

THEOREM 3.5. E is a completely continuous Fréchet differentiable map from $\underline{D}$ to $D, E^{\prime}$ is Lipschitz continuous, and $E^{\prime}(f)$ is injective.

Proof. Let $f \in \underline{D}$ and let $T=E(f) \in D$. By (3.3)

$$
T=-\alpha \mathcal{G}\left(T^{4}-f\right)
$$

and hence $T \in C^{2}[0, \tau] \cap D$. Therefore

$$
\left|T^{\prime \prime}\right| \leq 2 \alpha \Theta_{+}^{4}
$$

for all $\Theta \in D$. Hence $\mathcal{T}$ maps $D$ into the set

$$
\left\{T \in C^{2}[0, \tau]|| T^{\prime \prime} \mid \leq 2 \alpha \Theta_{+}^{4}\right\}
$$

which is precompact in $C[0, \tau]$.

We now show that $E$ is continuous. Let $f, g \in \underline{D}, U=E(f), V=E(g)$, and $S=V-U . S$ is a solution to the boundary value problem

$$
S^{\prime \prime}=\alpha(p S-(f-g)) ; S(0)=S(\tau)=0 .
$$

where

$$
0 \leq p=(V+U)\left(V^{2}+U^{2}\right) \leq 4 \Theta_{+}^{3} .
$$

Letting $D^{2}$ be the second derivative operator with homogeneous Dirichlet boundary conditions we have

$$
S=\left(-D^{2}+p\right)^{-1}(f-g)
$$

and therefore

$$
\|S\|_{2} \leq C\|f-g\|_{2}
$$


where $C=\left\|D^{-2}\right\|_{2}$ is independent of $f$ and $g$. We may multiply both sides of (3.9) by $S$ and integrate by parts to obtain

$$
\left\|S^{\prime}\right\|_{2} \leq \alpha\left(\|p\|_{\infty}\|S\|_{2}^{2}+\|S\|_{2}\|f-g\|_{2}\right) \leq \alpha\left(C+4 \Theta_{+}^{3} C^{2}\right)\|f-g\|_{2}^{2} .
$$

Since $S \in C^{2}[0, \tau], S$ is absolutely continuous and is the integral of its derivative. Hence for all $x \in[0, \tau]$

$$
S(x)=\int_{0}^{x} S^{\prime}(y) d y
$$

and

$$
|S(x)| \leq\left\|S^{\prime}\right\|_{2} \leq\left[\alpha\left(C+4 \Theta_{+}^{3} C^{2}\right)\right]^{1 / 2}\|f-g\|_{2} .
$$

Noting that

$$
\|f-g\|_{2} \leq \tau^{1 / 2}\|f-g\|_{\infty}
$$

completes the argument.

Finally we prove the differentiability assertions. One can compute the Gâteaux derivative $d E(f ; w)$ of $E$ at $f \in \underline{D}$ in the direction $w \in C[0, \tau]$ by inspection. $S=$ $d E(f ; w)$ is the solution of the boundary value problem

$$
S^{\prime \prime}=\alpha\left(4 E(f)^{3} S-w\right) ; S(0)=S(\tau)=0 .
$$

It is easy to see that $d E(f ; w)$ is continuous in $f$ and linear in $w$ and is therefore the Fréchet derivative. Moreover, one can see directly that $E^{\prime}(f)$ is injective for all $f \in \underline{D}$, for if $E^{\prime}(f) w=0$ the the solution $S$ of (3.10) vanishes and and $w=0$.

3.2. Properties of the map $F$. We will make use of the monotonicity properties of the solution $f=F(\Theta)$ of (2.2). The following lemma is a direct consequence of well known properties of the operator $\mathcal{K}$ [1].

Lemma 3.6. Let $c \in[0,1], \tau \in(0, \infty), \Theta_{l}, \Theta_{r} \geq 0$, and $\Theta \in D$, where $D$ is given by (3.2). Then the map $F$ that takes $\Theta$ to the solution of $(2.2)$ is a monotone function of $\Theta_{l}, \Theta_{r}, \Theta$ in the sense that if $\Sigma_{l} \leq \Theta_{l}, \Sigma_{r} \leq \Theta_{r}$, and $\Sigma \leq \Theta$ then

$$
F\left(\Sigma^{4}, \Sigma_{l}, \Sigma_{r}\right)(x) \leq F\left(\Theta^{4}, \Theta_{l}, \Theta_{r}\right)(x)
$$

for all $x \in[0, \tau]$. Moreover if $\Theta=\Theta_{l}=\Theta_{r}$, then $f(x)=\Theta^{4}$.

The consequence of Lemma 3.6 of most importance to us is

Corollary 3.7. Let $c \in[0,1], \tau \in(0, \infty), \Theta_{l}, \Theta_{r} \geq 0$, and $\Theta \in D$, where $D$ is given by (3.2). Then $f=F\left(\Theta^{4}\right)$ satisfies

$$
\Theta_{-}^{4} \leq f(x) \leq \Theta_{+}^{4} .
$$

Proof. The result follows from

$$
\Theta_{-}^{4}=F\left(\Theta_{-}, \Theta_{-}, \Theta_{-}\right) \leq F\left(\Theta, \Theta_{l}, \Theta_{r}\right) \leq F\left(\Theta_{+}, \Theta_{+}, \Theta_{+}\right)=\Theta_{+}^{4}
$$

as stated in the lemma. $\mathrm{Q}$

We close with a differentiability theorem.

THEOREM 3.8. The map $A(\Theta)=F\left(\Theta^{4}\right)$ is a Fréchet differentiable map from $D$ to $\underline{D}$. $A^{\prime}$ is Lipschitz continuous, and for all $w \in C[0, \tau]$,

$$
A^{\prime}(\Theta) w=4(I-c \mathcal{K})^{-1} \mathcal{K}\left(\Theta^{3} w\right)
$$


3.3. The map $\mathcal{T}$ and Existence of Solutions. Our main result in this section is that the map $\mathcal{T}$ is well defined and maps $D$ into itself. The complete continuity of $\mathcal{T}$ follows from that result. We begin with

Corollary 3.9. Let $c \in[0,1], \tau \in(0, \infty), \Theta_{l}, \Theta_{r} \geq 0$, and $\Theta \in D$, where $D$ is given by (3.2). Then (2.12) has a unique nonnegative solution $T=\mathcal{T}(\Theta)$ and $\mathcal{T}(\Theta) \in D$.

Proof. We apply Theorem 3.2 with $f=F\left(\Theta^{4}\right) \in \underline{D}$. $\mathrm{G}$

Existence of a solution to (2.13) will follow from the Schauder theorem and

Theorem 3.10. Let $c \in[0,1], \tau \in(0, \infty), \Theta_{l}, \Theta_{r} \geq 0$, and $D$ be given by (3.2). Then $\mathcal{T}$ is a completely continuous, Fréchet differentiable map from $D$ into itself and hence fixed point problem (2.13) has a solution $\Theta \in D$, which is also a solution of the system (1.1), (1.2), (1.3), (1.4).

Proof. The differentiability and complete continuity of $\mathcal{T}$ follow from Theorems 3.5 and 3.8. Since $\mathcal{T}(\Theta)=E\left(F\left(\Theta^{4}\right)\right)$, the existence follows from Theorem 3.5 and Lemma 3.6 and the Schauder fixed point theorem. $\square$

We will use the following monotonicity result for $\mathcal{T}$ in our uniqueness result. Corollary 3.3 and Lemma 3.6 together clearly imply

Lemma 3.11. Let $c \in[0,1], \tau \in(0, \infty)$, and $\Theta_{l}, \Theta_{r} \Sigma_{l}, \Sigma_{r} \geq 0$. Then if $\Sigma_{l} \leq \Theta_{l}$, $\Sigma_{r} \leq \Theta_{r}, 0 \leq \Sigma \leq \Theta$ then

$$
\mathcal{T}\left(\Sigma, \Sigma_{l}, \Sigma_{r}\right)(x) \leq \mathcal{T}\left(\Theta, \Theta_{l}, \Theta_{r}\right)(x)
$$

for all $x \in[0, \tau]$.

4. Uniqueness. In this section we address the uniqueness question. We show that $\mathcal{T}$ has a unique fixed point in $D$ and therefore the the system (1.1), (1.2), (1.3), (1.4) has a unique solution subject to the constraint that $\Theta \in D$.

We can use Lemma 3.11 in a direct way to prove our uniqueness result.

Theorem 4.1. Let $c \in[0,1], \tau \in(0, \infty), \Theta_{l}, \Theta_{r} \geq 0$. Then the solution to (2.12) (and hence of the system (1.1), (1.2), (1.3), (1.4)) is unique subject to the requirement that $\Theta \in D$.

Proof. Lemma 3.11 implies that the sequence defined by $U_{0}=\Theta_{-}$and for $n \geq 1$ by

$$
U_{n}=\mathcal{T}\left(U_{n-1}\right)
$$

is increasing and hence converges to a limit in $U \in L^{\infty}[0, \tau]$. Since the sequence $U_{n}$ lies in a compact subset of $C[0, \tau]$ by the complete continuity of $\mathcal{T}, U$ is continuous and $U=\mathcal{T}(U)$. Similarly the sequence defined by $V_{0}=\Theta_{+}$and for $n \geq 1$ by

$$
V_{n}=\mathcal{T}\left(V_{n-1}\right)
$$

is decreasing and converges to $V=\mathcal{T}(V) \in C[0,1]$. Moreover if $\Theta=\mathcal{T}(\Theta)$ then

$$
U \leq \Theta \leq V
$$

We complete the proof by showing that $U=V$. Using the first formulation as a compact fixed point problem we see that the difference $W=V-U \geq 0$ satisfies

$$
W^{\prime \prime}=\alpha(I-\mathcal{K})(I-c \mathcal{K})^{-1} p W ; W(0)=W(\tau)=0 .
$$

where $p=(V+U)\left(V^{2}+U^{2}\right) \geq 0$. 
Now, [1], $I-K$ is a self-adjoint positive definite operator in $L^{2}[0, \tau],\|\mathcal{K}\|_{L^{1}}<1$, and $(I-c \mathcal{K})^{-1}$ maps nonnegative functions to nonnegative functions. It is easy to check that $\mathcal{K}(1)(x) \leq 1$. Hence,

$$
\begin{aligned}
W^{\prime}(\tau)-W^{\prime}(0) & =\int_{0}^{\tau} W^{\prime \prime}(x) d x \\
& =\alpha \int_{0}^{\tau}(I-\mathcal{K})(1)(x)(I-c \mathcal{K})^{-1}(p W)(x) d x \geq 0 .
\end{aligned}
$$

Since $W \geq 0$ and $W(0)=W(\tau)=0$, we must have

$$
W^{\prime}(\tau)=W^{\prime}(0)=0 .
$$

Therefore (4.2) and the nonnegativity of $p W$ imply that

$$
\left\|(I-c \mathcal{K})^{-1} p W\right\|_{L^{1}} \leq\left\|\mathcal{K}(I-c \mathcal{K})^{-1} p W\right\|_{L^{1}},
$$

which, together with the fact that $\|\mathcal{K}\|_{L^{1}}<1$, implies that $W=0$.

Our final result concerns the dependence of $\Theta$ on the boundary data $\Theta_{l}, \Theta_{r}$. For a given $c \in[0,1], \tau \in(0, \infty)$, and $N_{c}>0$ we define $\Theta\left(x ; \Theta_{l}, \Theta_{r}\right)$ as the unique solution of (2.12) with boundary data $\Theta_{l}, \Theta_{r}$.

Our monotonicity result says that the solution is increasing in $\Theta_{l}$ and $\Theta_{r}$. The theorem follows directly from Lemma 3.6, Corollary 3.3.

Theorem 4.2. Let $c \in[0,1]$ and $\tau>0$ be given. Let $0 \leq \Sigma_{l} \leq \Theta_{l}$, and $0 \leq \Sigma_{r} \leq \Theta_{r}$. Then

$$
\Theta\left(x ; \Sigma_{l}, \Sigma_{r}\right) \leq \Theta\left(x ; \Theta_{l}, \Theta_{r}\right)
$$

for all $x \in[0, \tau]$.

Acknowledgments. The author thanks Jim Banoczi, Bob Martin, Necati Özışık, Fernando Reitich, and Chuck Siewert for many helpful conversations about this work. Some of the computations that lead to the theoretical results in this paper were done at the North Carolina Supercomputing Center.

\section{REFERENCES}

[1] I. W. Busbridge, The Mathematics of Radiative Transfer, no. 50 in Cambridge Tracts, Cambridge Univ. Press, Cambridge, 1960.

[2] S. Chandrasekhar, Radiative Transfer, Dover, New York, 1960.

[3] C. T. KELLEy, Multilevel source iteration accelerators for the linear transport equation in slab geometry, Tech. Report CRSC-TR93-5, North Carolina State University, Center for Research in Scientific Computation, April 1993. Trans. Th. Stat. Phys., to appear.

[4] E. W. LARSEN AND P. NELSON, Finite difference approximations and superconvergence for the discrete ordinate equations in slab geometry, SIAM J. Numer. Anal., 19 (1982), pp. 334-348.

[5] M. N. ÖzIşIK, Radiative Transfer and Interaction with Conduction and Convection, John Wiley and Sons, New York, 1973.

[6] R. H. Martin, Nonlinear Operators and Differential Equations in Banach Spaces, WileyInterscience, New York, 1976.

[7] J. PITKäranta AND R. SCOTT, Error estimates for the combined spatial and angular approximations of the transport equation in slab geometry, SIAM J. Numer. Anal., 20 (1983), pp. 922-950.

[8] C. E. SIEWERT, An improved iterative method for solving a class of coupled conductive-radiative heat transfer problems. Submitted for publication, 1994.

[9] C. E. Siewert and J. R. Thomas, A computational method for solving a class of coupled conductive-radiative heat transfer problems, J. Quant. Spectrosc. Radiat. Transfer, 45 (1991), pp. 273-281. 
A. Proof of (2.6). Following [2] we define for $x>0$

$$
E_{l}(x)=\int_{0}^{1} e^{-x / \nu} \frac{d \nu}{\nu^{l}}
$$

Note that

$$
\frac{d}{d x} E_{-1}=-E_{0} \text { and } \frac{d}{d x} E_{0}=-E_{1} .
$$

Let $q \in L^{1}([0, \tau])$. Define

$$
T_{l}(q)(x)=\int_{0}^{x} E_{0}(x-y) q(y) d y .
$$

Analyticity of $E_{0}$ in the right half plane, $E_{0}(0)=1$, and (A.2) imply that

$$
\frac{d T_{l}(q)(x)}{d x}=q(x)-\int_{0}^{x} E_{1}(x-y) q(y) d y .
$$

Similarly, if

$$
T_{r}(q)(x)=\int_{x}^{\tau} E_{0}(y-x) q(y) d y
$$

then

$$
\frac{d T_{r}(q)(x)}{d x}=-q(x)+\int_{x}^{\tau} E_{1}(y-x) q(y) d y .
$$

Set

$$
S=c f+(1-c) \Theta^{4} .
$$

For $\mu>0$ we integrate (1.1) forward in $x$, obtaining

$$
\psi(x, \mu)=\frac{1}{\mu} \int_{0}^{x} \exp (-(x-y) / \mu) S(y) d y+\exp (-x / \mu) \Theta_{l}^{4} .
$$

Similarly, for $\mu<0$, we integrate backwards to obtain,

$$
\begin{aligned}
\psi(x, \mu)= & -\frac{1}{\mu} \int_{x}^{\tau} \exp (-(x-y) / \mu) S(y) d y \\
& +\exp ((\tau-x) / \mu) \Theta_{r}^{4} .
\end{aligned}
$$

Using (A.5) and (A.6) we have

$$
\begin{aligned}
& \int_{0}^{1} \mu^{\prime} \psi\left(x, \mu^{\prime}\right) d \mu^{\prime}=\int_{0}^{x} E_{0}(x-y) S(y) d y+E_{-1}(x) \Theta_{l}^{4} \\
& \text { and } \\
& \int_{-1}^{0} \mu^{\prime} \psi\left(x, \mu^{\prime}\right) d \mu^{\prime}=-\int_{x}^{\tau} E_{0}(y-x) S(y) d y+E_{-1}(\tau-x) \Theta_{r}^{4} .
\end{aligned}
$$


We apply (A.3) and (A.4) to (A.7) and obtain

$$
\begin{aligned}
& \frac{d}{d x} \int_{0}^{1} \mu^{\prime} \psi\left(x, \mu^{\prime}\right) d \mu^{\prime}=S(x)-\int_{0}^{x} E_{1}(x-y) S(y) d y-E_{0}(x) \Theta_{l}^{4} \\
& \text { and } \\
& \frac{d}{d x} \int_{-1}^{0} \mu^{\prime} \psi\left(x, \mu^{\prime}\right) d \mu^{\prime}=S(x)-\int_{x}^{\tau} E_{1}(y-x) S(y) d y-E_{0}(\tau-x) \Theta_{r}^{4} .
\end{aligned}
$$

Adding the two equations in (A.8) gives

$$
\begin{aligned}
\frac{d}{d x} \int_{0}^{1} \mu^{\prime} \psi\left(x, \mu^{\prime}\right) d \mu^{\prime} & =2((I-\mathcal{K}) S)(x)-E_{0}(x) \Theta_{l}^{4}-E_{0}(\tau-x) \Theta_{r}^{4} \\
& =2(I-c \mathcal{K}) f(x)-2 g(x)+2(S(x)-f(x)) .
\end{aligned}
$$

Recalling that $(I-c \mathcal{K}) f(x)=g(x)$,

$$
S-f=(c-1) f+(1-c) \Theta^{4},
$$

and using (2.7) completes the proof. 